\title{
EUTOMIA
}

Revista de Literatura e Linguística

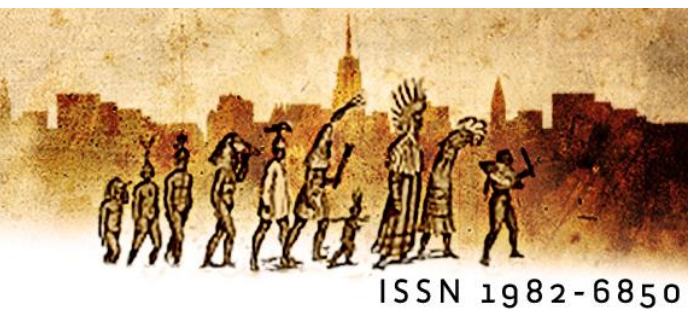

\section{L'éducation esthétique comme contribution au développement des émotions chez Vygotskij}

\section{Aesthetic education as a contribution for the development of emotions in Vygotskij}

\author{
Rita Hofstetter ' (Université de Genève) \\ Bernard Schneuwly (Université de Genève)
}

\begin{abstract}
Résumé : L'art et l'éducation esthétique sont des questions centrales dans le développement de l'émotion ou des sentiments au début de la vie active de Vygotskij dans les années 20. Or, il n'y revient plus guère dans son ouvre scientifique. La présente contribution décrit l'origine de son intérêt pour l'art et pour l'éduction esthétique et leur fonction et fonctionnement dans ses premiers écrit. Puis il montre que certes Vygotskij aborde la question de l'imagination comme base de l'activité créatrice, mais sans revenir sur le rôle de l'art et de l'éducation esthétique dans le développement des émotions. La contribution termine par la présentation d'une possible explication de l'énigme de la disparition de l'art dans l'œuvre scientifique de Vygotskij.
\end{abstract}

Mots-clés: Education esthétique ; développement ; émotion ; sentiments.

Resumo: A arte e a educação estética são temas centrais no desenvolvimento da emoção ou dos sentimentos no início da vida ativa de Vygotskij nos anos 20. Mas, ele nunca mais retomou isso no seu trabalho científico. A presente contribuição descreve a origem de seu interesse pela arte e pela educação estética e sua função e funcionamento nos primeiros escritos. Em seguida, ele mostra que Vygotskij certamente aborda a questão da imaginação como base da atividade criativa, mas sem retornar ao papel da arte e da educação estética no desenvolvimento das emoções. A contribuição termina com a apresentação de uma possível explicação do enigma do desaparecimento da arte na obra científica de Vygotskij.

Palavras-chave: Educação estética; desenvolvimento; emoção; sentimentos.

La question des émotions est présente dans l'œuvre de Vygotskij depuis ses premiers écrits à la fin des années 1920. Tout autant l'est depuis le début son intérêt pour l'éducation et l'enseignement comme moteur du développement. Et pourtant, le rapport 
entre enseignement et développement, au cœur du chapitre 6 de Pensée et langage n'aborde pas la question de l'émotion, son développement et son éducation. Pourtant, des réflexions à ce propos sont menées dans ses deux premiers livres quand il discute la fonction de l'art. Puis, à partir de 1928 ce thème disparaît presque complètement ${ }^{1}$ Certes, Vygotskij discute le rôle de l'imagination en lien avec l'émotion et analyse l'activité créatrice chez l'élève, mais il ne systématise pas le rôle de l'enseignement et en ne reprend pas du tout la question de la fonction de l'art, de la «réaction esthétique » pourtant au cœur de ses préoccupations dans sa théorie de l'art. Le présent texte a pour objectif de d'exposer cette énigme de la disparition le plus précisément possible. Pour ce faire nous montrons dans un premier temps d'où vient l'intérêt de Vygotskij pour l'enseignement et la fonction de l'art ; puis nous décrivons sa théorie de la fonction de l'art d'une part, le rôle de l'éducation esthétique pour que tout un chacun ait accès à l'art et sa réception d'autre part. En décrivant ensuite le travail de Vygotskij sur l'imagination et l'activité créatrice nous montrons que la question de la réception d'œuvres et plus généralement de la fonction de l'art disparaît presque totalement. Nous terminerons par quelques hypothèses pour expliquer cette disparition.

Dans notre travail, nous tenons compte de la totalité des textes disponibles de Vygotski où il a abordé cette problématique de 1920 à $1934{ }^{2}$ en nous référant aussi à l'immense littérature secondaire ${ }^{3}$.

\section{Les origines de la pensée éducative et de la théorie de l’art de Vygotskij ${ }^{4}$}

\footnotetext{
${ }^{1} \mathrm{~F}$. Sève fait le même constat: «Or, chose étonnante, Vygotski n'est jamais revenu sur ces questions esthétiques dans son abondante œuvre ultérieure, si l'on excepte un article rédigé en 1932- ». (2005, p. 9)

${ }^{2}$ Nous nous référons aux originaux russes et aux différentes traductions à disposition : français, allemand, anglais, italien, espagnol et portugais, selon les cas.

${ }^{3}$ Nous nous sommes inspirés pour ce faire de la remarque de Kozulin : "Vygotsky's œuvre has all the qualities of a superior text that is rich, dense and open enough to serve as a critical reflection of twentieth century psychology». (Kozulin, 1990, p. 3)

${ }^{4}$ Les connaissances dont nous disposons sur la première période de la vie active de Vygotski permettent de constater que'il pose d'emblée des problèmes de psychologie et d'éducation contrairement au "Cinderella type account» encore très répandu. Voir notamment Van der Veer et Valsiner, 1991; Dobkin, 1982; Yaroshevsky, 1989; Vygodskaja et Lifanova, 2000; Zavershneva, 2010, 2016; Vygotsky Notebooks, 1912$1934 / 2018$.
} 
Pour comprendre, voire accentuer, le paradoxe que nous venons d'énoncer, tentons de saisir ce qui amène Vygotski à écrire ses premiers ouvrages qui constituent les embryons de la théorie qu'il développera tout au long de son activité scientifique. ${ }^{5}$

Au moment même où débute le nouveau régime en URSS en 1917, Vygotski parachève ses études de droit d'une part, de littérature et lettres de l'autre, et retourne à Gomel, la ville de son enfance. L'occupation allemande y perdure jusqu'en janvier 1919 et le gouvernement de la ville ne sera formé qu'en avril. L'une de ses tâches principales: construire un nouveau système scolaire, les enseignants en place refusant leur collaboration. Vygotski y participe de manière active en enseignant dans plusieurs écoles. Quand est fondé le "technicum pédagogique ", une sorte d'école normale, il contribue presque d'emblée au collectif pédagogique qu'il administre. Vygotski dispense des cours de psychologie générale de l'enfant, expérimentale et pédagogique. Il concourt aussi à la formation continue des enseignants, notamment des écoles rurales, en donnant des cours de psychologie pédagogique. II propose de créer un cabinet scolaire de psychologie expérimentale qui a pour but de montrer aux futurs enseignants des expériences psychologiques, d'impulser des travaux pratiques et des recherches, d'analyser l'activité enseignante. Vygotski y réalise moult expériences.

Parallèlement, il déploie une importante activité culturelle : il rédige des dizaines de recensions d'ouvrages et de critiques de théâtres et ballets ${ }^{6}$ et publie même un petit livre sur l'artiste Bykhovsky ${ }^{7}$; il édite une revue littéraire et fonde une maison d'édition d'ouvrages littéraires; il donne des dizaines de conférences sur des écrivains classiques et contemporains, mais aussi sur la théorie de la relativité ou la psychanalyse. Vygotski se distingue encore comme organisateur de représentations théâtrales invitant à Gomel les troupes les plus innovantes de l'URSS qui s'inspirent des théories de mise en scène aussi bien de Stanislavski que de Meyerhold et de Tairov. Vygotski participe de surcroît à la direction du Musée local de la presse, qui met à disposition des lecteurs des dizaines de quotidiens et organise des soirées littéraires avec des écrivains contemporains.

\footnotetext{
${ }^{5}$ C'est ainsi du moins qu'on peut les lire en connaissant la suite de son travail; voir Kozulin, qui montre les influences majeures de Hegel, Potebnja, Freud, etc. (1990). Voir aussi Van der Veer, (1984).

${ }^{6}$ On trouve dans l'article de Sobkin et Mazanov (2015) une critique d'un ballet par Vygotski qui montre la présence en germe de nombreuses idées et concepts qu'il approfondit plus tard.

${ }^{7}$ https://www.russianartandbooks.com/cgi-bin/russianart/02841R.html. Van der Veer (2015) présente une vue d'ensemble des critiques de théâtre ; Sobkin (2016) en propose une analyse très approfondie.
} 
Cette intense activité au service de la construction de l'école et des institutions culturelles, couplée avec la publication de textes de critiques littéraires et théâtrales, se matérialise dans deux ouvrages majeurs: Psychologie de l'art (1925/2005) et Psychologie pédagogique (1926/2006). Ils constituent les premières esquisses de sa future conception de l'éducation, et notamment du rôle de l'éducation dans le domaine de l'imagination et de l'émotion. Le premier est centré sur l'exploration de la fonction de l'art et le second sur la pédagogie et l'enseignement. Signalons que la plupart des biographes du Vygotski de cette période (voir note 3 ) insistent surtout sur ses connaissances et inscriptions dans les milieux artistiques, en particulier littéraires, et mettent en évidence ses filiations philosophiques, évoquant notamment la place de Hegel, Spinoza, parfois aussi de Marx (voir aussi Védrines et Schneuwly 2021). Quant à nous, nous supposons que l'activité professionnelle de Vygotski comme enseignant et formateur est tout aussi déterminante dans l'orientation de ses travaux. Nous défendrions volontiers la thèse que la psychologie était un moyen pour lui de saisir quel rôle l'éducation joue dans le développement et quels sont les mécanismes qui permettent de comprendre l'effet des œuvres humaines sur la constitution de la personnalité. La psychologie pourrait s'avérer une sorte de détour pour atteindre d'autres buts. Kozulin (1990) l'énonce d'une certaine manière: «Vygotsky was primarily a thinker, psychology being just the most appropriate stage on which the drama of ideas could be played». (p. 2$)^{8}$

\section{Psychologie de l'art: l'art comme technique sociale des sentiments exige une éducation de la perception de l’art}

Cette dense activité dans les milieux artistiques, couplée d'observations, analyses, cours, recensions, ${ }^{9}$ amène Vygotski à aborder plus systématiquement la question à laquelle répond sa Psychologie de l'artı: "La question est donc: est-il possible d'établir de lois

\footnotetext{
${ }^{8}$ Il n'est pas inintéressant de faire un parallèle avec Piaget, autre grand psychologue du développement, qui, lui aussi, fait de la psychologie par détour pour résoudre d'autres questions, en l'occurrence épistémologiques. Il n'est pas exclu que le fait même de venir pour ainsi dire de l'extérieur de la discipline permette d'aborder les questions d'un autre point de vue ; un phénomène connu en histoire des sciences.

${ }^{9}$ Van der Veer en donne une liste impressionnante (2007). Marques analyse le Vygotskij inconnu à travers ses écrits sur l'art de 19115 à 1925 .

${ }^{10}$ Le nombre de textes commentant cet ouvrage est substantiel. Pour ce volet, nous avons plus particulièrement utilisé, outre Kozulin, Van der Veer et Valsiner (1991) ; Kellog (s.d.). West, 1999; Guimaraes Lima (1995).
} 
psychologiques de l'action de l'art sur l'homme ou cela s'avère-t-il impossible?» (1925/2005, p. 41)

Rejetant à la fois des méthodes qui cherchent une solution à ce problème dans la psychologie de l'artiste, du lecteur ou du récepteur, Vygotski propose une démarche indirecte, s'apparentant à celle de l'historien qui doit construire la connaissance du passé par de multiples sources dont aucune ne correspond au réel du passé, ou à celle du zoologiste qui doit reconstituer la structure d'un animal sur la base de quelques os : partir de la spécificité des œuvres d'art. ${ }^{11}$ Dans Psychologie de l'art, Vygotski (1925/2005) avance que l'une des dimensions centrales est le mode essentiellement ambivalent de l'expérience esthétique qui peut contenir aussi bien, pour prendre cet exemple, l'horreur d'un événement décrit qu'un sentiment de libération que produit l'œuvre comme tout. La réaction du lecteur réside dans cette expérience contradictoire. Cette dialectique se manifeste dans une catharsis, présupposant la participation active du lecteur, qui permet une décharge d'affect, restructurant, grâce à l'imagination, l'expérience interne. Transposant cette fonction de l'art et ses lois de fonctionnement au niveau de la société, Vygotski peut alors affirmer:

Nous devons convenir que la science ne contamine pas simplement toute la société avec les idées d'un seul, pas plus que la technique ne prolonge simplement le bras de l'homme, de la même façon l'art est en quelque sorte un "sentiment social» prolongé, ou " une technique des sentiments » $(\text { p. } 338)^{12}$

Deux éléments essentiels apparaissent dans cette conception de l'art. Premièrement, l'art est une technique, un outil ou un instrument qui agit sur des sentiments. Cette idée d'instrument sera systématisée ultérieurement par Vygotski qui l'appliquera à d'autres dimensions de la vie psychique. Mais la portée de cette phrase est autre encore, comme en témoignent ces lignes: "L'art est plutôt une organisation de notre comportement pour

\footnotetext{
${ }^{11}$ A ce propos, Vygotskij écrit dans une note datant de 1926: «The method of The Psychology of Art is analysis, abstraction (that is why there is not a word about the fable as such; I ignore its specific traits); let the historian show which feelings were experienced in which eras and in what form.» (1922-1934/2018, p. 76).

${ }^{12}$ Vygotsky, Psychology of art, 244. Une autre métaphore de Vygotksij: «Art is vaccination, i.e., overcompensation (in every sense of the word: poison + immunity), i.e., weakness that creates strength, disease that generates super-health, and previously it was considered as just disease. But is the vaccination with smallpox really a vaccination of disease rather than health? It is both, but the meaning of disease is in super-health. This is the central complex of ideas that I adopted in the Psychology of art, and not Freud, not the formalists. At bottom, this is a dialectical principle of a philosophical epistemological nature.» (1922$1934 / 2018$, p. 76 )
} 
l'avenir, une orientation vers l'avant, une exigence qui peut-être ne sera jamais satisfaite mais qui nous force à aspirer par-delà notre vie à ce qu'il y a au-delà. " C'est une manière spécifique d'agir : «Tout ce que fait l'art, il le fait dans notre corps et par son intermédiaire ». Et plus généralement: "Tout ce dont il est question dans ce chapitre, c'est que toute la portée pratique de l'art se ramène enfin de compte a son action éducative. » (p. 352).

Cette action éducative, cependant, est elle-même médiatisée ; son chemin doit être frayé : via les discours sociaux à son propos, et notamment la critique de l'œuvre d'art, via l'éducation elle-même. L'éducation, notamment l'enseignement, facilite l'action éducative de l'art. Contre des prises de position de certains artistes ou pédagogues extraire l'art du giron scolaire en vertu de la soi-disant exceptionnalité de l'art comme pratique humaine, Vygotski défend l'absolve nécessité de la médiation scolaire par deux démarches : celle de la construction de connaissances de l'œuvre du point de vue historique et social ; celle de I'analyse et la connaissance de son fonctionnement esthétique afin d'éviter toute réduction de l'œuvre à son contenu. L'explication qui permet une forme de conscience réflexive, loin d'empêcher l'émotion, oriente l'effet de l'œuvre, le fortifie, le préserve, confère une direction au déploiement des processus « inconscients » qu'il éveille.

Nous trouvons ici l'un de nos fils conducteurs: l'éducation comme transmission culturelle. Elle est ici, de fait, dédoublée : I'action éducative de l'art présuppose l'éducation à I'art. Mais les quelques éléments fournis dans la Psychologie de l'art, ouvrage qui vise, nous l'avons vu, à répondre à la question de comment l'art agit sur l'être humain, ne sont que brièvement esquissés. Dans cet ouvrage c'est avant tout le Vygotski critique de l'art qui s'exprime, réfléchissant notamment sur sa propre pratique de critique, sur la fonction sociale de son activité dans le domaine de sa diffusion sous toutes ses formes.

Vygotski reviendra brièvement sur la question de la fonction de l'art dans un court texte qui apparaît à la fois comme le résumé des thèses principales de Psychologie de l'art et comme l'esquisse d'un programme de recherche à venir :

The infectious nature of art is not based on a simple transmission of feelings from one to another-in this sense, the speech of the speaker, the cry of pain, the loud hurrah are no less infectious. Verses on sadness lift us above the sadness, they defeat it, overcome it, solve it. How they achieve this, by what psychological means-that is the $\mathrm{X}$, the proper name of art, the unknown quantity from which any investigation should start. (1927/201, p. 115) 
Comme nous le verrons, cette recherche sur le $\mathrm{X}$ ne se concrétisera pas.

\section{Éducation esthétique comme éducation dédoublée : éducation à l'art qui est action éducative $^{13}$}

La question de la médiation par l'art et, ici surtout de la médiation de l'art par l'éducation et l'enseignement, est approfondie dans l'ouvrage que Vygotski écrit dans le contexte de son activité de formateur. Psychology of education (1926/2005) est un manuel pour la formation des enseignants, issu sans doute des cours que Vygotski leur a dispensés ${ }^{14}$. Le chapitre sur "L'éducation esthétique » (1926/sous presse) débute par une analyse approfondie de la place en général attribuée à l'esthétique à l'école : elle est au service de la pédagogie ; elle n'a pas de fonction éducative pour elle-même et à travers elle-même. Dans cette perspective critique, Vygotski distingue trois fonctions pédagogiques externes à éviter. La première est l'éducation morale: dans ce domaine, l'esthétique devrait avoir un effet immédiat, résultant de l'analyse de l'œuvre de ce point de vue. Confisquée, la dimension esthétique des œuvres disparaît. Considérer les œuvres comme reflet de la réalité, deuxième fonction pédagogique, est tout aussi problématique: les personnages représenteraient une sorte de photographie des groupes sociaux de leur époque ; ceci non seulement donne une fausse idée de la réalité, mais fait disparaître également les éléments esthétiques des œuvres lues qui se réduisent ainsi à leur contenu. Et troisièmement, l'œuvre d'art est abordée comme une fin en soi ; le sens de l'expérience esthétique se limite à la perception de plaisir qu'éprouve l'enfant, abstraction faite de la spécificité de l'œuvre d'art.

Ces tendances pédagogiques dominantes de l'éducation esthétique que Vygotski critique sont notamment le résultat d'une conception psychologique qui considère la perception esthétique comme une expérience passive. L'émotion esthétique au contraire est le résultat d'une réaction esthétique active, postule Vygotski. Théorisant cette réaction, il s'oppose là aussi à des conceptions classiques, répandues notamment dans les mouvements de l'éducation nouvelle. ${ }^{15}$ L'émotion esthétique ne consiste pas en une

\footnotetext{
${ }^{13}$ Moins connu, ce chapitre a également fait l'objet d'un certain nombre d'interprétations et commentaires, notamment: Frois, 2010; Sawaia, et da Silva, 2015:343-360; Clerc-Georgy, A., 2016 ; Brossard (s.d.). Dans aucun de ces textes cependant, l'accent n'est mis sur la dimension enseignement tel qu'envisagé par Vygotski.

${ }^{14}$ Jentzen (2019) situe les cours donnés par Vygotskij ayant abouti à l'ouvrage à 1921-1923.

15 Tout en s'inspirant de démarches développées au sein de ce mouvement, Vygotski s'oppose fondamentalement à la conception rousseauiste de l'enfant qui y domine, notamment à propos de l'art et de l'imagination. Pour une présentation détaillée, voir Hofstetter et Schneuwly (2009).
} 
intensification de la vie, une mise en commun d'un sentiment ressenti par un auteur, une « infection » par le sentiment d'un artiste, comme le formule Tolstoï. C'est la catharsis, la résolution de contradictions vécues dans la perception d'une œuvre qui créent l'effet. Pour ce faire, toute personne doit en quelque sorte recréer l'œuvre, ou du moins récapituler pour partie le processus créatif à la base de l'œuvre. "L'art porte en soi une telle émotion dialectique qui, en restructurant le comportement, implique toujours une lutte interne complexe qui se résout dans la catharsis. » (p. 140) Autrement dit : la réaction esthétique implique une participation active, un travail de l'imagination. Elle a pour point de référence les dimensions esthétiques qui donnent forme à la matière. C'est à ce type de réception d'œuvres esthétiques auquel il convient éduquer, estime Vygotski: ni l'art comme éducation étroitement morale, ni l'art comme représentation du réel, ni l'art pour l'art, mais l'art comme possibilité de transformation de la personne par la catharsis. Plus généralement encore :

II suffit de se pencher même brièvement sur la réaction esthétique pour remarquer que son objectif final $n^{\prime}$ est pas de reproduire une quelconque réaction réelle, mais de la dépasser et de la vaincre [...] Ainsi, l'art n'est pas la reproduction de la vie, mais le moyen par lequel l'humain surpasse la vie. (p. 135-136)

Il s'agit là d'une exigence extrêmement forte qui implique une conception sophistiquée et multidimensionnelle de l'éducation esthétique. Vygotski la théorise en distinguant trois dimensions séparées, mais interreliées, que cette éducation doit affronter : a) développer la créativité de l'élève; b) développer des capacités et connaissances techniques liées aux œuvres d'art et à la pratique esthétique; c) former au jugement esthétique, enseigner la capacité d'appréhender l'œuvre d'art. (p. 139)

Le développement des capacités de création esthétique des élèves a pour effet de transformer le lien profond entre expérience vécue et émotion et de permettre son extériorisation en lui donnant une forme matérielle sémiotique. Le dessin et la rédaction de textes littéraires notamment permettent à l'enfant de développer ses capacités créatrices tout en transformant son rapport à son expérience et à ses émotions. Nous verrons que c'est cette dimension de l'éducation esthétique que seule Vygotskij développera plus en détail.

L'éducation esthétique comprend ensuite un enseignement à des capacités techniques. Cette formation doit être large, avoir trait à toutes les formes d'art et se rapporter à la fois 
aux productions des élèves eux-mêmes et à la réception des œuvres : seul un enseignement qui dépasse les techniques en tant que telles prend sens dans une éducation esthétique générale.

C'est cette dernière forme d'éducation, l'appréhension des œuvres, la plus complexe, qui est de loin la moins développée, regrette Vygotski. II s'agit pourtant de la plus importante :

C'est pourquoi, quand on parle d'éducation esthétique dans le système d'enseignement général, il faut toujours avoir en tête d'intégrer l'enfant à l'expérience esthétique humaine : l'amener à un art magistral et solide et, à travers lui, inclure le psychisme de l'enfant dans ce travail universel commun qu'a réalisé l'humanité depuis des milliers d'années pour sublimer par l'art son propre psychisme. Voilà le but et l'objectif principal. (p. 144)

Vygotski termine ces quelques remarques sur l'éducation esthétique par une prise de position ferme en faveur de la nécessité de donner accès à l'art à tous. L'idée répandue qu'il faudrait une éducation différente pour les doués et pour les «moins doués " doit être combattue, affirme-t-il. Le don n'est pas un point de départ, mais un but de l'éducation ${ }^{16}$.

Le potentiel de création de chacun d'entre nous est de devenir le complice de Shakespeare dans ses tragédies et celui de Beethoven dans ses symphonies, c'est le meilleur indicateur du fait que nous avons du Shakespeare et du Beethoven en nous. (p. 151)

\section{Imagination et activité créative : disparition de la réflexion sur l'art et l'éducation à l'art}

L'art apparaît ainsi comme technique, comme instrument psychologique pour agir sur des processus psychiques, plus particulièrement sur les émotions. Vygotski généralisera plus tard cette idée que des moyens sémiotiques sont des instruments pour agir sur les processus psychiques: la mémoire, l'attention, la perception, la langue elle-même. II systématisera l'analogie, que nous avons déjà rencontrée, entre les instruments qui permettent d'agir sur le monde matériel au sens large du terme et, par leur appropriation, sur ceux qui les utilisent; et les instruments qui agissent sur les fonctions psychiques, celles des autres et les siennes propres : les moyens sémiotiques au sens large, les signes. En tant qu'instruments psychologiques, les signes transforment fondamentalement ces processus:

\footnotetext{
${ }^{16}$ Cette prise de position anticipe le fameux article que L. Sève (1964) « Les dons n'existent pas » dans lequel cet auteur développe en détail cette question essentielle.
} 
l'écriture, la manière de produire le langage ; les concepts, la manière de penser ; les cartes, la manière de percevoir l'espace; etc. L'appropriation de ces instruments sémiotiques élaborés au cours de l'histoire et leur intériorisation à la fois réorganisent les fonctions psychiques, les articulent avec d'autres et constituent simultanément la base pour construire de nouvelles fonctions, en fait de nouveaux systèmes psychiques. Parmi la liste des instruments sémiotiques énumérés par Vygotski figurent «les œuvres d'art» (1930/1985). Et pourtant, contrairement aux nombreux autres instruments psychologiques qu'il étudiera ultérieurement (par exemple dans le chapitre 6 de Pensée et langage), les œuvres d'art ne font plus l'objet d'analyse approfondie ; ou, marginalement, dans son petit ouvrage sur Imagination et activité créatrice 1930/sous presse) et, d'une manière indirecte, dans son esquisse d'une psychologie de l'acteur. (1932/1999).

Dans le chapitre 2 Imagination et activité créatrice, consacré à «Imagination et réalité ", Vygotski revient brièvement sur la fonction de l'art. II reprend explicitement la distinction entre outils techniques et outils psychologiques :

En fin de compte, à quoi sert une œuvre artistique? N'a-t-elle pas une influence sur notre monde intérieur, sur nos pensées et nos sentiments aussi clairement que les outils techniques en ont sur le monde extérieur, sur l'univers de la nature? (p. 21)

Sans se référer explicitement à son ouvrage Psychologie de l'art ${ }^{17}$, Vygotski en rappelle néanmoins la substance: les œuvres d'art ont une logique interne issue de la combinaison complexe entre éléments internes de l'auteur, notamment pensées et émotions, et le monde extérieur. L'œuvre constitue l'extériorisation, l'incarnation extérieure, la «cristallisation » écrit-il, de cette transformation créatrice du matériau, et forme en quelque sorte la fin d'une boucle qui part de l'extérieur, des événements, de la réalité multiforme, pour y revenir. Ce bref rappel de la fonction instrumentale de l'art (Vygotski mentionne la prose, poésie, musique), le seul dans l'ouvrage, permet d'en déduire que sa conception fondamentale de l'art n'a pas changé. Mais elle n'a pas non plus été approfondie à la lumière de la systématisation de son approche théorique, notamment de la place centrale attribuée à l'instrument, et plus généralement de l'importance des œuvres

\footnotetext{
${ }^{17}$ Vygotski ne fait en effet pas référence à son ouvrage Psychologie de l'art, certes non publié de son vivant pour des raisons inconnues (voir Vygodskaja \& Lifanova (1999; pp. 69-70); il ne réfère jamais non plus au texte publié «Contemporary Psychology and Art» (1927/2011) qui en résume des éléments essentiels.
} 
humaines comme moyens de transformer au fil de I'histoire leur propre fonctionnement psychique.

Ce bref rappel lui sert essentiellement à contraster la fonction des œuvres d'art avec les productions de l'imagination et de l'activité créatrice de l'enfant dont l'analyse est au cœur du texte. Vygotski oppose explicitement les deux types de création d'artistes et d'enfants et contredit ainsi l'interprétation convenue dans le sillon des idées rousseauistes de l'enfant et de l'éducation que nous avons déjà évoquées plus haut.

Dans les chapitres 6, 7 et 8 de l'ouvrage, Vygotski analyse les activités créatrices en écriture, théâtre et dessin et leur fonction dans le développement. Mais il insiste clairement sur le fait que ces activités sont loin de donner lieu à des œuvres d'art. D'une part, parce qu'il s'agit de productions caractérisées par un certain syncrétisme : les genres d'activités ne sont pas distingués et maîtrisés en tant que tels par les enfants; et plus généralement même, l'enfant exprime de manière immédiate son vécu, ses actions et pensées, par exemple lorsqu'il dessine et met en scène. D'autre part, l'enfant n'a pas coupé les liens avec des intérêts et des expériences personnels. A la fois originales, de par précisément leur origine, et stéréotypées, par défaut aussi de maîtrise des moyens artistiques, I'enfant utilise de fait des schémas. Productions enfantines d'un moment, elles n'ont aucune prétention ni fonction comparable à des œuvres d'art. Elles ont pour fonction et effet précisément de développer l'imagination, d'agir sur les émotions, d'entrer en contact et maîtriser des formes rudimentaires des langages artistiques - où l'on retrouve, très brièvement, la question de la réception.

Cette conception des résultats de l'activité créatrice des enfants est fondamentalement différente des thèses répandues à l'époque, notamment par Tolstoï. Certes, si l'on repère parfois des trouvailles étonnantes dans les textes d'enfants que Tolstoï n'hésite pas à comparer aux plus belles pages de Goethe lui-même, elles se situent toujours dans un contexte étroit, réalisant des formes limitées, voire primitives. Cette manière de voir a pour conséquence de ne pas considérer l'intervention éducative comme nuisant à la spontanéité naturelle de l'enfant, tuant sa créativité, dans la visée rousseauiste défendue par Tolstoï, mais au contraire comme la condition nécessaire de cette créativité. L'action de l'éducateur, par exemple celle de Tolstoï, est, de fait, massive, et Vygotski l'analyse, admiratif, en en montrant les rouages et l'influence. Dans l'idéologie «doctrinairereligieuse » d'un Tolstoï, et d'autres personnes adhérant à la même conception, estime 
Vygotski, cette action éducative constitue un point aveugle. Elle est pourtant la condition même de la possibilité de développer l'imagination et l'activité créatrice. La maîtrise technique notamment, à laquelle l'enseignement peut contribuer, joue un rôle essentiel dans l'appropriation et le développement des capacités d'écriture et de dessin.

Tout art cultivant des moyens spécifiques pour matérialiser ses objets possède sa propre technique et l'association des disciplines techniques aux pratiques artistiques est probablement ce qu'un pédagogue peut offrir de plus précieux, à cet âge-là. (p. 96)

\section{Un étonnant silence - Tentative d'explication}

Le livre sur l'imagination et l'activité créatrice vise à comprendre la construction des capacités humaines, notamment à travers l'éducation et l'enseignement dans le domaine d'activités artistiques diverses. ${ }^{18}$ II n'est donc pas étonnant que la question de la réception n'y soit pas abordée. Mais elle ne l'est pas non plus dans d'autres contextes qui s'y prêteraient pourtant parfaitement. Notamment dans le fameux chapitre 6 de Pensée et langage qui aborde systématiquement la question de la transformation de processus psychiques par l'enseignement de contenus liés à des disciplines scolaires: langues étrangères, grammaire, écriture, concepts scientifiques. Rappelons-en la thèse principale : la transmission systématique de savoirs et savoir-faire organisés en disciplines scolaires est la condition de la transformation du rapport à des savoirs et savoir-faire spontanés, quotidiens. La conscientisation des fonctions par leur explicitation dans la pensée verbale transforme fondamentalement les fonctions qui se réorganisent et vont entretenir de nouveaux rapports avec d'autres fonctions (ce qui ne signifie nullement que tout devient conscient) formant de nouveaux systèmes psychiques. On reconnaît sans peine, en filigrane, quelques éléments développés par Vygotski aussi bien à propos de l'éducation esthétique dans Psychologie pédagogique que, plus subtilement et de manière plus approfondie, dans Psychologie de l'art. Ce qui sans doute lui tient le plus à cœur, l'effet « éducatif » de l'art, sa fonction de "technique sociale du sentiment », qui présuppose sa médiation, n'apparaît même pas en pointillé dans ses travaux ultérieurs dans lesquels Vygotski introduit le

\footnotetext{
${ }^{18}$ Notons que Vygotski insiste par ailleurs sur le fait que le développement des capacités créatives se limite trop souvent à l'art : « Malheureusement, l'éducation traditionnelle qui éloignait les enfants du travail ne leur permettait généralement d'exprimer et de développer leurs capacités créatrices que dans le domaine de l'art. » (p. 97).
} 
concept heuristique d'instrument psychologique et approfondit sa théorie désormais bien connue du rapport entre enseignement et développement. Comment expliquer cela?

Rappelons que l'art est défini comme technique sociale des sentiments. Et rappelons par ailleurs que Vygotski, à plusieurs reprises, s'attaque à la construction d'une théorie des émotions : il le mentionne dans le texte sur les systèmes psychiques; et il produit un long manuscrit, inachevé, intitulé La doctrine concernant les émotions: études historicopsychologiques ${ }^{19}$ dans lequel il prépare le matériau pour produire une théorie. ${ }^{20}$ Deux thèses principales y sont discutées et démontrées.

1. Toutes les théories existantes sur l'émotion sont profondément dualistes affirme Vygotskij. ${ }^{21}$ Soit elles cherchent un substrat corporel de l'émotion et doivent recourir à des explications métaphysiques pour aborder des émotions plus complexes; soit elles définissent les émotions comme phénomènes spirituels sans possibilité de les rattacher aux manifestations corporelles. Contrairement à ce que peuvent affirmer les tenants eux-mêmes de la première position (notamment James et Lange), cette dualité n'est pas issue de la philosophie moniste de Spinoza, mais reproduit l'explication cartésienne des émotions avec notamment la domination possible des émotions par la volonté.

2. La seule issue possible, esquissée par Vygotski dans le manuscrit, est d'analyser les émotions comme produit d'un développement réorganisant les rapports entre les différentes fonctions psychiques, dans la lignée de ce qu'il décrit minutieusement dans ses travaux pédologiques (1931-34/2018). Processus psychiques et physiques s'interpénètrent profondément, transformant les lois mêmes du développement,

\footnotetext{
${ }^{19}$ En russe учеине peut se traduire par doctrine, enseignement, apprentissage. Le sous-titre est essentiel: en fait il s'agit d'une étude fouillée de la doctrine dominante des émotions, analogue à la «critique de l'économie politique» qui préparait et accompagnait l'élaboration du Capital. Vygtoski n'a pas pu écrire ce Capital et ne fait, en guise de proposition, que quelques références à Spinoza en contrepoint à la critique.

${ }^{20}$ On trouve certaines thèses dans le cours « Les émotions et leur développement chez l'enfant» (1932/2011). Se référant à d'autres recherches, Akhutina (2003, p. 185) estime qu'après la période de l'élaboration des systèmes psychiques, qui dépasse la théorie des fonctions psychiques supérieures, Vygotski s'est concentré, dans ses deux dernières années de vie, sur la question de la connexion entre affect et intellect. (Akhutina, 2003. ${ }^{21}$ La revendication de la filiation de Damasio (2003) avec Spinoza ressemble à cet égard à celle que revendiquent James et Lange dont Vygotski montre sur des dizaines de pages qu'elle est illégitime.Une analyse approfondie suivant la démarche de Vygotski permettrait probablement d'arriver à la conclusion qu'il est de fait cartésien; ce que montre par exemple Kambouchner (2010) dans son texte "L'erreur de Damasio » (qui cependant ne traite qu'insuffisamment la différence entre Descartes et Spinoza concernant la théorie des émotions). Magiolino et Smolka (2013) laissent également entendre que la revendication de Damasio est peu fondée et proposent par ailleurs une voie pour avancer sur la question des émotions à travers les concepts vygotskiens de signification et de drame. Voir aussi Ratner (2000) pour une réinterprétation du cadre de Vygotski.
} 
constituant de nouveaux systèmes psychiques. Les instruments sociohistoriquement constitués sont les conditions rendant ces transformations possibles. Encore faut-il que de tels outils soient produits, permettant la transformation des émotions. Les cultures, dans l'histoire et par l'histoire, construisent, transforment, élaborent sans cesse des émotions particulières grâce à des techniques sociales. Et Vygotski de constater que l'art joue le rôle de révélateur et d'instrument de transformation des émotions :

La différence entre l'adoration mystique vouée par le chevalier à sa dame et la galanterie des nobles du XVIle siècle reste inexpliquée [...] En effet on ne peut admettre que la simple perception d'une silhouette féminine provoque automatiquement un nombre infini de réactions organiques d'où pourrait naître un amour comme celui de Dante pour Béatrice si l'on ne présuppose pas l'ensemble des idées théologiques, politiques, esthétiques, scientifiques qui composent la conscience du génial Alighieri.» $(1925 / 2005)^{22}$

Le manuscrit La doctrine concernant les émotions: études historico-psychologiques se limite pour l'essentiel à la critique des théories existantes; Vygotski situe le problème, élabore du matériau pour construire une théorie des émotions qui réponde aux exigences découlant de la critique sévère formulée à l'encontre des propositions existantes. C'est sans doute précisément l'impossibilité de formuler une théorie des émotions qui l'empêche d'articuler son intuition de la fonction de l'art comme technique sociale des sentiments avec ses analyses du développement des fonctions psychiques supérieures et la création et transformation de systèmes psychiques nouveaux. Le « $X$ » dont il parle dans son texte sur I'art contemporain (voir ci-dessus) restera « X».

\section{Conclusions}

\footnotetext{
${ }^{22}$ Le développement récent, intense, d'un champ de recherche dédié à l'histoire de l'émotion (Bouquet \& Nagy, 2016) confirme en tout point l'intuition profonde de Vygotski. Dans une synthèse de travaux, Sère (2017) note: "Les émotions sont des phénomènes culturels et non pas des données biologiques. Mais le biologique existe pourtant dans la manifestation de l'émotion. D'où l'impression que l'histoire des émotions peut désormais, après les années d'expérimentation, tourner le dos à la psychologie cognitive et autres sciences neurobiologiques pour traiter des émotions historiquement. » (p. 128). Voir Sander (2017) pour une présentation succincte des approches actuelles qui fait référence à une lignée de philosophes, à l'exception notoire de Spinoza...
} 
Nous avons défini un double fil conducteur pour explorer certains aspects de l'œuvre de Vygotskij : a) l'art est un moyen d'éducation; il permet notamment d'éduquer les sentiments, les émotions; b) la fonction éducative au sens large de l'art implique une éducation à l'art. Nous avons trouvé en effet chez Vygotski une théorie de l'art et de l'éducation esthétique qui met au centre la question du travail sur les émotions. L'œuvre artistique est une "technique sociale du sentiment ", énonce-t-il, en analysant la logique interne des œuvres transformant les émotions. Encore faut-il garantir une éducation à la réception des œuvres d'art, condition pour que leur action éducative de l'art puisse se déployer; et cette éducation doit s'adresser à toutes et tous dans la société égalitaire que I'URSS de l'époque a, à son avis, comme projet politique d'instaurer. Comme nous l'avons évoqué plus haut, l'éducation esthétique pour la réception d'œuvres d'art est ébauchée dans le premier livre publié par Vygotski Psychology of education, mais ne sera cependant pas étayée. II concentrera plus tard ses efforts sur des descriptions plus précises de la construction de l'imagination et de l'activité créatrice à travers l'activité artistique de l'enfant qui a pour dessein non pas la création d'œuvres, mais précisément celle de développer ces fonctions en lien avec un travail sur les émotions. La question de l'effet de l'art et ses mécanismes, tout comme celle de l'éducation à la réception n'est plus abordée.

Esquisse d'éducation esthétique donc qui attendait sans doute, pour son achèvement, une théorie des émotions permettant de définir plus précisément les possibilités d'éducation à l'art comme action éducative ; théorie qui, elle aussi est restée en l'état d'ébauche, avec un accent fort sur la nécessité de pouvoir analyser les émotions comme produit historique et culturel grâce notamment à l'action éducative de l'art.

\section{Références bibliographiques}

AKHUTINA, T. V. L.S. Vygotsky and A.R. Luria: Foundations of Neuropsychology. Journal of Russian and East European Psychology, 41, p. 159-190, 2003.

BOUQUET, D. \& HAGY, P. Pour une histoire intellectuelle des émotions. L'Atelier du Centre de recherches historiques, 16 (en ligne). BROSSARD, M. (s.d.). Vygotski et le problème de l'enseignement esthétique. Manuscrit non publié, 2016.

CLERC-GEORGY, A. L'imagination dans le développement de la créativité et de I'apprentissage. In : CAPRON-PUOZZO, I. (org.). La créativité en éducation et formation. Bruxelles : De Boeck, 2016, p. 79-92. 
DAMASIO, A.R. Spinoza avait raison. Joie et tristesse, le cerveau des émotions. Paris : Odile Jacob, 2003.

DOBKIN, S. Ages dans Days (Semyon Dobkin's Reminiscences). In: LEVITIN, K. (ed.), One is not Born a Personality. Profiles of Soviet Education Psychologists. Moscow: Progress Publishers, 1982, pp. 23-38.

FROIS, J.P. Lev Vygotsky's theory of aesthetic experience. In: CONSTATIONO, T; WHITE, B. (eds.). Essays on aesthetic education for the 21st century. London: Sage, 2010, p. 109-122.

GUIMARAES LIMA, M. From Aesthetics to Psychology: Notes on Vygotsky's "Psychology of Art". Anthropology \& Education Quarterly, 26, p. 410-424, 1995.

HOFSTETTER, R.; SCHNEUWLY, B. Knowledge for teaching and knowledge to teach: two contrasting figures of New Education: Claparède and Vygotsky. Paedagogica Historica. International Journal of the History of Education, 45(4\&5), p. 605-629, 2009.

JANTZEN, W. Lev Semënovič Vygotskij als Wissenschaftler und Begründer der kulturhistorischen Theorie. In: JANTZEN, W; RICHTER, G. (eds.). Kulturhistorische Psychologie und Methodologie. Arbeiten zur humanwissenschaftlichen Umgestaltung der Psychologie. Berlin: Lehmans Media, 2019, p. 193-220.

KAMBOUCHNER, D. Emotions et raison chez Descartes. L'erreur de Damasio. Pensando Revista de Filosofia, 1, p. 30-51, 2010.

KELLOG, D. (s.d.). The Real Ideal. An illustrated guide to Vygotsky's Psychology of Art. Seoul: Manuscrit non publié. Disponível em : $<$ http://lchc.ucsd.edu/mca/Paper/The Real Ideal.pdf $>$.

KOZULIN, A. Vygotsky's Psychology. A Biography of Ideas. New York: Harvester, 1990.

MAGIOLINO, L. L. S.; SMOLKA, A. L. B. How Do Emotions Signify? Social Relations and Psychological Functions in the Dramatic Constitution of Subjects. Mind, Culture, and Activity, 20, p. 96-112, 2013.

MAROUES, P. N. O Vygótski incógnito: escritos sobre arte (1915-1926) (Thèse de doctorat non publiée). São Paulo: Universidade de São Paulo, 2015.

RATNER, C. A Cultural-Psychological Analysis of Emotions. Culture \& Psychology, 6(1), p. 5-39, 2000.

SANDER, D.; HERSCHDORFER, N. Préface. In: Emotions. Salenstein: Benteli, 2017, p. 6-9.

SAWAIA, B. B.; Silva, D. N. H. Pelo reencantamento da psicologia: em busca da positividade epistemológica da imaginação e da emoção no desenvolvimento humano. Cadernos Cedes. 35, p. 343-360, 2015. 
SERE, B. Histoire des émotions : I'heure des synthèses ". Revue de l'histoire des religions, 1, p. 119-132, 2017.

SEVE, F. Présentation. In : VYGOTSKI, L. S. Psychologie de l'art. Paris: La Dispute, 2005, p. 7-11.

SÈVE, L. Les "dons" n'existent pas. L'École et la Nation, 132, p. 39-64, 1964.

SOBKIN, V.S. L.S. Vygotsky and the Theater. Journal of Russian \& East European Psychology, 53, p. 1-92, 2016.

SOBKIN, V.S.; MAZANOV, V.S. Comments on L.S. Vygotsky's Theater Review on E.V. Gel'tser's Performance». Journal of Russian \& East European Psychology, 52, p. 128-148, 2015 .

VAN DER VEER, R. Early periods in the work of L.S. Vygotski: the influence of Spinoza. In: HEDEGAARD, M.; HAKKARAINEN, P.; ENGESTRÖM, Y. (eds.). Learning and Teaching on a Scientific Basis. Aarhus: Psyckologisk Institut, 1984, p. 87-98.

VAN DER VEER, R. Vygotsky in context: 1900 - 1935. In: DANIELS, H.; COLE, M.; WERTSCH, J.V. (eds.). The Cambridge Companion to Vygotsky. Cambridge: Cambridge University Press, 2007, p. 21-49.

VAN DER VEER, R. Vygotsky, the theater critic: 1922-1923. History of Human Sciences, 28, p. $102-110,2015$.

VAN DER VEER, R.; VALSINER, J. Understanding Vygotsky. A quest for synthesis. Oxford: Blackwell, 1991.

VYGODSKAJA, G. L.; LIFANOVA, T. M. Lev Semjonovič Vygotskij. Leben - Tätigkeit Persönlichkeit. Hamburg: Kovač, 2000.

VYGOTSKY, L. S. Psychologie de l'art. Paris : La Dispute, 1925/2005.

VYGOTSKIJ, L.S. Pedagogičeskaja psihologija [Psychologie pédagogique]. Moskva: Astrel', 1926/2005.

VYGOTSKY, L. S. Éducation esthétique. In: Imagination. Textes choisis. Berne: Lang, 1926/sous presse.

VYGOTSKY, L. S. Contemporary Psychology and Art. Journal of Aesthetic Education, 45, p. 107-117, (1927/2011).

VYGOTSKY, L. S. La méthode instrumentale. In: SCHNEUWLY, B.; BRONCKART, J.P. (eds.). Vygotsky aujourd'hui. Neuchâtel: Delachaux et Niestlé, 1930/1935, p. 39-47. 
VYGOTSKY, L. S. Imagination et activité créatrice. In : Imagination. Textes choisis. Berne: Lang, 1930/sous presse.

VYGOTSKY, L. S. On the Problem of the Psychology of the Actor's Creative Work. Collected Works of L.S. Vygotsky. New York: Routledge, v. 6, p. 237-244, 1932/1999.

VYGOTSKY, L.S. La science du développement de l'enfant. Textes pédologiques (19311934). Berne : Lang, 1931-34/2018.

VYGOTSKY, L. S. Les émotions et leur développement chez l'enfant. In: Leçons de psychologie. Paris: La Dispute, 1932/2011, p. 123-155.

VYGOTSKY, L. S. Théorie des émotions. Etude historico-psychologie. Paris: L'Harmattan, $1933 / 1998$.

WEST, D. W. Lev Vygotsky's Psychology of Art and Literature. Changing English, 6(1), p. 47-55, 1999. DOI: 10.1080/1358684990060105.

YAROSHEVSKY, M. Lev Vygotsky. Moscow: Progress Publisher, 1989.

ZAVERSHNEVA, E. lu. The Vygotsky Family Archive (1912-1934). Journal of Russian \& East European Psychology, 48(1), p. 14-33, 2010.

ZAVERSHNEVA, E. lu. Vygotsky the unpublished: an overview of the personal archive (1912-1934.). In: YASNITSKY, A.; VAN DER VEER, R. (eds.). Revisionist Revolution in Vygotsky Studies. London: Routledge, 2016, p. 94-125.

ZAVERSHNEVA, E. Iu.; VAN DER VEER, R. Vygotsky's Notebooks. A Selection. Berlin: Springer, 1922-1934/2018.

\footnotetext{
${ }^{\text {i }}$ Professeure ordinaire en histoire de l'éducation de I'Université de Genève, Faculté de psychologie et sciences de l'éducation. Équipe de Recherche en Histoire Sociale de l'Éducation (ERHISE).

E-mail: Rita.Hofstetter@unige.ch

ii Professeur honoraire de l'Université de Genève- Faculté de psychologie et sciences de l'éducation. Équipe de Recherche en Histoire Sociale de l'Éducation (ERHISE) et Groupe de recherche pour l'analyse du français enseigné (GRAFE).

E-mail: Bernard.Schneuwly@unige.ch
} 